\title{
Contrasting associations of insulin resistance with diabetes, cardiovascular disease and all-cause mortality in the elderly: PROSPER long-term follow-up
}

\author{
Paul Welsh • David Preiss • Suzanne M. Lloyd • Anton J. de Craen • \\ J. Wouter Jukema • Rudi G. Westendorp • Brendan M. Buckley • \\ Patricia M. Kearney • Andrew Briggs • David J. Stott • Ian Ford • Naveed Sattar
}

Received: 17 June 2014 / Accepted: 29 August 2014 / Published online: 30 September 2014

(C) Springer-Verlag Berlin Heidelberg 2014

\begin{abstract}
Aims/hypothesis Insulin resistance is commonly proposed as a precursor to both type 2 diabetes and cardiovascular disease (CVD), yet few studies have directly compared insulin resistance with both outcomes simultaneously and determined whether associations with each outcome differ in strength or are comparable. We assessed the association of fasting insulin and HOMA-IR with incident CVD and diabetes in older people.

Methods In the long-term follow-up of the Prospective Study of Pravastatin in the Elderly at Risk (PROSPER) cohort, HOMA-IR measurement was available in 4,742 older people (70-82 years) without diabetes at baseline. Of these, 283 developed diabetes during the 3.2 year within-trial followup, while 1,943 all-cause deaths, 470 CHD deaths (identified from death records) and 590 fatal/non-fatal CVD events
\end{abstract}

Electronic supplementary material The online version of this article (doi:10.1007/s00125-014-3383-9) contains peer-reviewed but unedited supplementary material, which is available to authorised users.

P. Welsh $(\varangle) \cdot$ D. Preiss $\cdot$ N. Sattar $(\square)$

British Heart Foundation Glasgow Cardiovascular Research Centre,

University of Glasgow, Glasgow G12 8TA, UK

e-mail: Paul.Welsh@glasgow.ac.uk

e-mail: Naveed.Sattar@glasgow.ac.uk

S. M. Lloyd · I. Ford

Robertson Centre for Biostatistics, University of Glasgow, Glasgow, UK

A. J. de Craen • R. G. Westendorp

Department of Gerontology and Geriatrics, Leiden University

Medical Centre, Leiden, The Netherlands

J. W. Jukema

Department of Cardiology, Leiden University Medical Centre,

Leiden, The Netherlands (identified from medical record linkage in the Scottish participants) occurred during an extended 8.6 years of total followup. Cause-specific Cox proportional-hazards models were fitted using multivariable models.

Results Higher HOMA-IR was associated with incident diabetes: HR 4.80 (95\% CI 3.14, 7.33) comparing extreme thirds after adjustment for confounders. However, HOMA-IR in the top third was not associated with all-cause mortality, CHD mortality or fatal/non-fatal CVD: HR 1.02 (95\% CI 0.90 , $1.17), 1.03(0.79,1.36)$ and $0.94(0.74,1.20)$, respectively. Results were similar when fasting insulin was considered as an exposure.

Conclusions/interpretation Our data support insulin resistance as a predictor of diabetes in later life but, perhaps surprisingly, suggest this pathway is of negligible importance to CVD outcomes in the elderly.

\section{B. M. Buckley \\ Department of Pharmacology and Therapeutics, Cork University Hospital, Cork, Ireland \\ P. M. Kearney \\ Department of Epidemiology \& Public Health, University College Cork, Cork, Ireland}

\section{A. Briggs}

Institute of Health \& Wellbeing, University of Glasgow, Glasgow, UK

D. J. Stott

Institute of Cardiovascular and Medical Sciences, University of Glasgow, Glasgow, UK 
Keywords All-cause mortality - Cardiovascular disease . Insulin resistance $\cdot$ Risk factors

\author{
Abbreviations \\ CVD Cardiovascular disease \\ PROSPER Prospective Study of Pravastatin in the Elderly \\ at Risk trial
}

\section{Introduction}

The 'common soil' hypothesis of cardiovascular disease (CVD) and diabetes has given rise to widely adopted terms such as 'metabolic syndrome' and 'cardiometabolic risk factors'. While it is clear that diabetes is one of the strongest CVD risk factors [1], the extent to which moderate metabolic perturbations in advance of diabetes are associated with CVD appears weaker than previously appreciated [2-5].

It has been suggested that insulin resistance and its consequential compensating hyperinsulinaemia may be important steps in CVD pathogenesis. The possibility that hyperinsulinaemia promotes atherosclerosis is tempered by observations that insulin can be protective in some animal models [6], although insulin resistance itself is usually considered an adverse CVD phenotype. For instance, insulin resistance may act through inflammatory or endothelial dysfunction pathways to promote atherosclerosis [7]. Recent meta-analyses suggest the association of HOMA-IR and fasting insulin with CVD is likely to be modest, although many individual studies had limited power [8, 9]; for example, the most powerful study relating HOMA-IR to CVD had only 318 events during a 30 year follow-up [9]. Furthermore, the association of insulin resistance with CVD risk in the elderly, one of the most important groups in terms of current and future public health and population CVD burden, remains unclear because of the lack of relevant studies.

Using the Prospective Study of Pravastatin in the Elderly at Risk (PROSPER) trial cohort, we aimed to investigate the association of HOMA-IR with CVD and all-cause mortality in older people. HOMA-IR was also related to risk of in-trial diabetes as a secondary endpoint to verify that HOMA-IR was measuring propensity to develop diabetes and to compare and contrast HOMA-IR associations with diabetes versus vascular outcomes.
Fig. 1 Flow diagram for inclusion in PROSPER and data availability for the present study. $N$ refers to number for fasting insulin exposure, and $n$ refers to number for HOMA-IR exposure. ACM, all-cause mortality

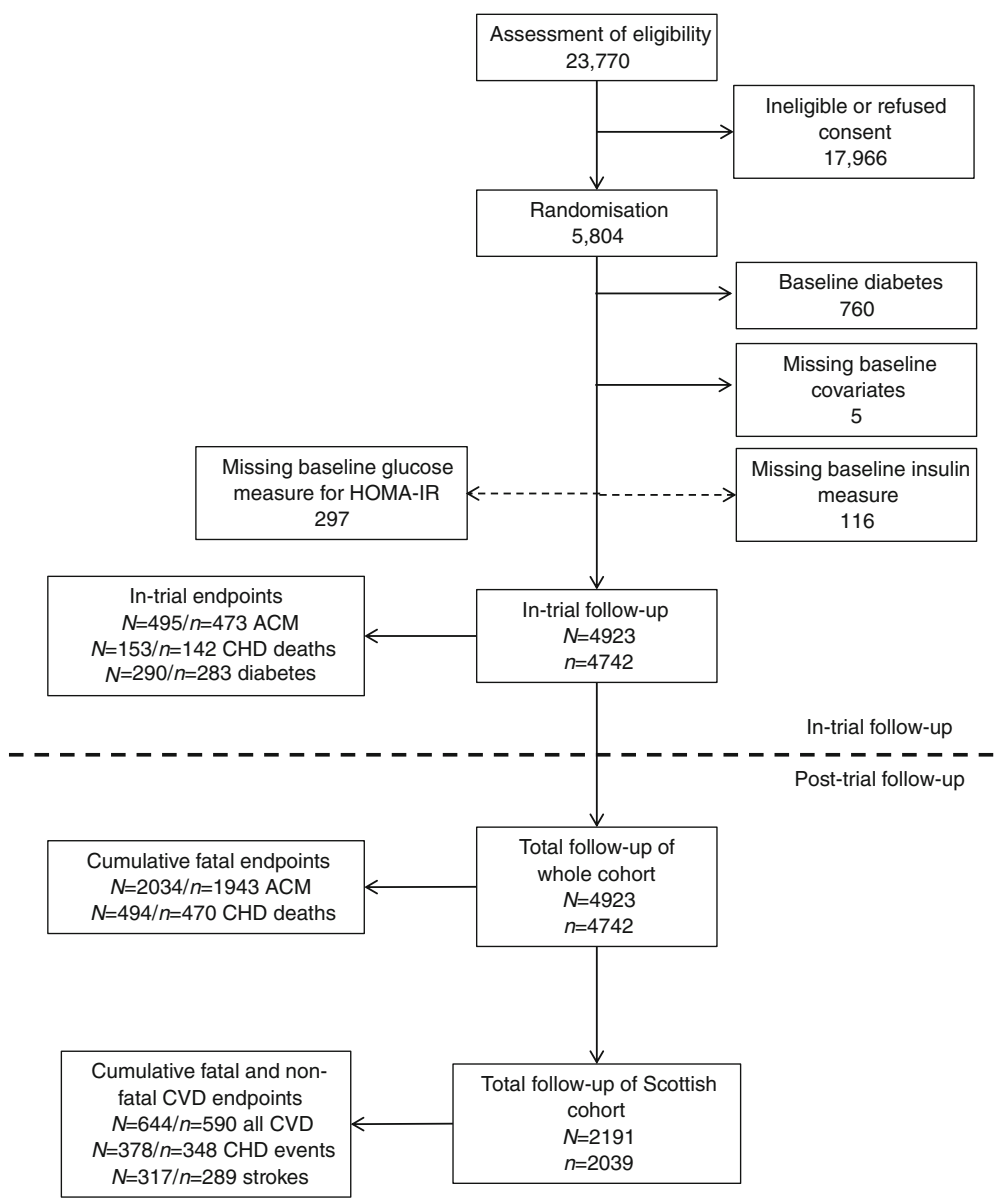




\section{Methods}

Participants PROSPER was a randomised placebocontrolled trial designed to investigate the effect of pravastatin $40 \mathrm{mg}$ on cardiovascular events in elderly participants (aged $70-82$ years) with pre-existing or raised risk of CVD. Details of the original trial (3.2 years follow-up) and the long-term follow-up of PROSPER have been published $[10,11]$. In brief, between December 1997 and May 1999 a total of 5,804 individuals were screened and enrolled in Scotland, Ireland and the Netherlands. Participants were asked to attend study visits after an overnight fast, venepuncture was performed, and separated plasma samples were stored at $-80^{\circ} \mathrm{C}$ until assay. The institutional ethics review boards of all centres approved the protocol, and all participants gave written informed consent.

The present study excluded 760 participants with diabetes at baseline; cases of both known diabetes based on self-report and/or taking of oral hypoglycaemic agents and undiagnosed diabetes based on baseline glucose measurements (fasting blood glucose concentration $\geq 7.0 \mathrm{mmol} / 1$ or a glucose concentration of $\geq 11.1 \mathrm{mmol} / 1$ when fasting status was uncertain $[<5 \%])$ were excluded.

The primary outcome of the original trial was the composite endpoint of CVD, which included death from CHD, nonfatal myocardial infarction, and fatal or non-fatal stroke. All within-trial CVD events were adjudicated by a blinded independent review board $[10,12]$. New-onset type 2 diabetes was diagnosed in the trial on the basis of a single elevated fasting plasma glucose result $(\geq 7.0 \mathrm{mmol} / \mathrm{l})$ or starting glucoselowering therapy reported during in-trial study visits. The fatal endpoints presented in this analysis are based on events recorded during in-trial follow-up and then entirely by computerised record linkage during post-trial follow-up over an average of 8.6 years of total follow-up [11]. Incident diabetes over the extended follow-up is not available.

For Scottish participants, we were able to link to hospital discharge summaries held by the Information Services Division of the National Health Service for Scotland by means of established record-linkage methods as previously

Table 1 Baseline CVD risk factors by thirds of HOMA-IR

\begin{tabular}{|c|c|c|c|c|c|}
\hline \multirow[t]{2}{*}{ Baseline characteristic } & \multirow[t]{2}{*}{ Level } & \multicolumn{3}{|c|}{ Thirds of HOMA-IR } & \multirow[t]{2}{*}{$p$ value } \\
\hline & & $\begin{array}{l}\mathrm{T} 1 \\
\leq 1.37 \\
(N=1,766)\end{array}$ & $\begin{array}{l}\mathrm{T} 2 \\
1.38-2.39 \\
(N=1,675)\end{array}$ & $\begin{array}{l}\mathrm{T} 3 \\
\geq 2.40 \\
(N=1,301)\end{array}$ & \\
\hline Treatment allocation & Placebo & $883(50.0 \%)$ & $845(50.4 \%)$ & $650(50.0 \%)$ & 0.95 \\
\hline \multirow[t]{3}{*}{ Country } & Scotland & $668(37.8 \%)$ & $768(45.9 \%)$ & $603(46.3 \%)$ & \multirow[t]{3}{*}{$<0.0001$} \\
\hline & Ireland & $823(46.6 \%)$ & $586(35.0 \%)$ & $448(34.4 \%)$ & \\
\hline & Netherlands & $275(15.6 \%)$ & $321(19.2 \%)$ & $250(19.2 \%)$ & \\
\hline Age & Years & $75.43(3.37)$ & $75.36(3.38)$ & $75.31(3.34)$ & 0.63 \\
\hline Sex & Female & $892(50.5 \%)$ & $892(53.3 \%)$ & $735(56.5 \%)$ & 0.0045 \\
\hline Current smoker & Yes & $643(36.4 \%)$ & $434(25.9 \%)$ & $262(20.1 \%)$ & $<0.0001$ \\
\hline BMI & $\mathrm{kg} / \mathrm{m}^{2}$ & $24.50(3.35)$ & $26.99(3.60)$ & $29.12(4.19)$ & $<0.0001$ \\
\hline SBP & $\mathrm{mmHg}$ & $152.8(22.4)$ & $154.5(21.1)$ & $155.2(21.6)$ & 0.0079 \\
\hline DBP & $\mathrm{mmHg}$ & $82.6(11.5)$ & $84.2(11.3)$ & $84.3(11.2)$ & $<0.0001$ \\
\hline HDL-cholesterol & $\mathrm{mmol} / \mathrm{l}$ & $1.41(0.37)$ & $1.28(0.33)$ & $1.17(0.31)$ & $<0.0001$ \\
\hline LDL-cholesterol & $\mathrm{mmol} / 1$ & $3.80(0.82)$ & $3.88(0.80)$ & $3.79(0.78)$ & 0.0037 \\
\hline Triacylglycerol & Log mmol/1 & $0.16(0.36)$ & $0.34(0.38)$ & $0.54(0.41)$ & $<0.0001$ \\
\hline History of hypertension & Yes & $985(55.8 \%)$ & $1,088(65.0 \%)$ & $900(69.2 \%)$ & $<0.0001$ \\
\hline History of CHD & Yes & $558(31.6 \%)$ & $556(33.2 \%)$ & $448(34.4 \%)$ & 0.25 \\
\hline History of CVD & Yes & $200(11.3 \%)$ & $203(12.1 \%)$ & $149(11.5 \%)$ & 0.74 \\
\hline History of peripheral vascular disease & Yes & $207(11.7 \%)$ & $180(10.7 \%)$ & $140(10.8 \%)$ & 0.59 \\
\hline Diuretic use & Yes & $610(34.5 \%)$ & $733(43.8 \%)$ & $663(51.0 \%)$ & $<0.0001$ \\
\hline ACE inhibitor use & Yes & $229(13.0 \%)$ & $270(16.1 \%)$ & $229(17.6 \%)$ & 0.0011 \\
\hline ARB use & Yes & $33(1.9 \%)$ & $25(1.5 \%)$ & $32(2.5 \%)$ & 0.16 \\
\hline$\beta$-blocker use & Yes & $428(24.2 \%)$ & $448(26.7 \%)$ & $371(28.5 \%)$ & 0.0253 \\
\hline CCB use & Yes & $365(20.7 \%)$ & $464(27.7 \%)$ & $355(27.3 \%)$ & 0.0001 \\
\hline
\end{tabular}

Values in parentheses are percentages or standard deviations

$\mathrm{ARB}$, angiotensin receptor blockers; $\mathrm{CCB}$, calcium channel blocker 
described, thereby obtaining information on non-fatal CVD events [11]. For both deaths and hospital discharge summaries, data were available until 30 June 2008. Hence, for the analysis of composite fatal or non-fatal cardiovascular outcomes, our cohort is restricted to Scottish participants $(n=$ 2,191 for the insulin analyses and $n=2,039$ for HOMA-IR analyses) (Fig. 1).

Lipids and glucose were measured as previously reported [10-13]. Insulin was measured using a commercially available ultrasensitive assay (Mercodia; Diagenics, Milton Keynes, UK) or, if the results were in excess of the limit of detection $(140 \mathrm{pmol} / \mathrm{l})$, by a standard sensitivity assay from the same manufacturer $(n=65)$. The high-sensitivity assay had an intra-assay CV of $2.6 \%$ and an interassay $\mathrm{CV}$ of $6.2 \%$, and the normal sensitivity assay had an intraassay $\mathrm{CV}$ of $3.8 \%$ and an inter-assay $\mathrm{CV}$ of $7.2 \%$. The exposure of interest in the present study was either fasting insulin or HOMA-IR calculated as: fasting plasma glucose $(\mathrm{mmol} / \mathrm{l}) \times$ insulin $(\mathrm{pmol} / \mathrm{l}) / 135$.

Statistical methods Baseline characteristics were summarised as mean (SD) for continuous variables and as count (\%) for categorical variables. Because of the non-normal distribution of insulin and HOMA-IR, logarithmic transformations were applied to these variables. The distribution of fasting insulin and HOMA-IR in the cohort was split into thirds, and related to risk factors of interest across the distribution using ANOVA. For the present study, only the first event was included as an endpoint for a given model (i.e. in the all CVD model, deaths preceded by a non-fatal event were excluded and participants were censored at the date of the non- fatal event). Years of follow-up were calculated from the date of randomisation until censoring due to end of the follow-up period, withdrawal of consent or death, whichever came first. Cause-specific Cox proportional-hazards models were fitted; we investigated both minimally and more fully adjusted models. In addition to the insulin or HOMA-IR variable, the minimally adjusted models also adjusted for the design variables of country and treatment randomisation (pravastatin or placebo). The more fully adjusted models further adjusted for relevant baseline risk factors (country, randomised treatment, age, sex, smoking status, BMI, systolic blood pressure, diastolic blood pressure, HDL-cholesterol and LDL-cholesterol, $\log$ triacylglycerol histories of CVD, medications that may affect HOMA-IR, diuretics, ACE inhibitors, angiotensin receptor blockers, $\beta$-blockers, calcium channel blockers). Tests for interaction were undertaken (ESM Methods). All statistical analyses were conducted in SAS version 9.2

\section{Results}

Among 5,044 participants without diabetes at baseline, data were available in 4,923 (97.6\%) for fasting insulin and in 4,742 (94.0\%) for HOMA-IR (Fig. 1).

HOMA-IR was positively associated with BMI and systolic and diastolic blood pressure, and was inversely associated with HDL-cholesterol. Women and non-smokers had higher levels of HOMA-IR. HOMA-IR was higher among participants from Scotland and the Netherlands, but there was no association with age or history of CVD (Table 1). Results for

Table 2 Association of fasting insulin and HOMA-IR with incident diabetes during in-trial follow-up

\begin{tabular}{|c|c|c|c|c|c|c|c|c|}
\hline \multirow[t]{2}{*}{ Variable/level } & \multirow[t]{2}{*}{$N$ participants } & \multirow[t]{2}{*}{$n$ events } & \multicolumn{3}{|c|}{ Model 1} & \multicolumn{3}{|c|}{ Model 2} \\
\hline & & & $\mathrm{HR}$ & $95 \% \mathrm{CI}$ & $p$ value & HR & $95 \% \mathrm{CI}$ & $p$ value \\
\hline $\begin{array}{l}\text { HOMA-IR } \\
\text { (per SD increase) }\end{array}$ & 4,742 & 283 & 1.92 & $1.74,2.13$ & $<0.0001$ & 1.69 & $1.50,1.91$ & $<0.0001$ \\
\hline HOMA-IR T1 & 1,766 & 32 & Ref & - & & Ref & - & \\
\hline HOMA-IR T2 & 1,675 & 92 & 3.09 & $2.06,4.62$ & & 2.54 & $1.68,3.85$ & \\
\hline HOMA-IR T3 & 1,301 & 159 & 7.11 & $4.86,10.4$ & $<0.0001$ & 4.80 & $3.14,7.33$ & $<0.0001$ \\
\hline $\begin{array}{l}\text { Insulin } \\
\text { (per SD increase) }\end{array}$ & 4,923 & 290 & 1.62 & $1.48,1.78$ & $<0.0001$ & 1.37 & $1.21,1.54$ & $<0.0001$ \\
\hline Insulin T1 & 1,745 & 43 & Ref & - & & Ref & - & \\
\hline Insulin T2 & 1,674 & 96 & 2.36 & $1.65,3.39$ & & 1.81 & $1.25,2.63$ & \\
\hline Insulin T3 & 1,504 & 151 & 4.19 & $2.99,5.88$ & $<0.0001$ & 2.43 & $1.65,3.58$ & $<0.0001$ \\
\hline
\end{tabular}

$p$ value is for test for linear trend in HR estimates (across distribution)

Model 1 adjusted for country, randomised treatment

Model 2 additionally adjusted for age, sex, smoking status, BMI, systolic blood pressure, diastolic blood pressure, HDL-cholesterol, LDL-cholesterol, $\log$ triacylglycerols, histories of hypertension, coronary, cerebrovascular and peripheral vascular disease, diuretics, ACE inhibitors, angiotensin receptor blockers, $\beta$-blockers and calcium channel blockers

T1, lower third of the distribution; T2, middle third of the distribution; T3, top third of the distribution 
fasting insulin were similar (ESM Table 1). HOMA-IR and fasting insulin were very strongly correlated $(r=0.959$, $p<0.0001$ ).

Associations of fasting insulin and HOMA-IR with risk of developing diabetes Over 3.2 years of within-trial followup, 283 participants were diagnosed with diabetes. Both HOMA-IR and fasting insulin were strongly associated with incident diabetes before and after adjustment for CVD risk factors and BMI (Table 2). In adjusted analyses, those in the highest third of HOMA-IR and fasting insulin were at 4.80 $(95 \%$ CI 3.14, 7.33$)$ and 2.43 (95\% CI 1.65, 3.58) higher risk of developing diabetes, respectively (both $p<0.0001$ ). There was no strong evidence of interaction (ESM Fig. 1).

Associations of fasting insulin and HOMA-IR with risk of CVD events and mortality Over the 8.6 years of follow-up, there were 1,943 all-cause mortality events. In continuous models only adjusting for randomised treatment and country, both HOMA-IR $(p=0.01)$ and fasting insulin $(p=0.015)$ showed trends towards an inverse association with all-cause mortality, although these associations were attenuated to the null on further adjustment for other risk factors (Table 3). For CHD mortality and all CVD (fatal and non-fatal), neither

Table 3 Association of HOMA-IR and fasting insulin with all-cause mortality and CVD outcomes during total follow-up

\begin{tabular}{|c|c|c|c|c|c|c|c|c|c|}
\hline \multirow[t]{2}{*}{ Endpoint } & \multirow[t]{2}{*}{ Variable/level } & \multirow[t]{2}{*}{$N$ participants } & \multirow[t]{2}{*}{$n$ events } & \multicolumn{3}{|c|}{ Model 1} & \multicolumn{3}{|c|}{ Model 2} \\
\hline & & & & HR & $95 \% \mathrm{CI}$ & $p$ value & HR & $95 \% \mathrm{CI}$ & $p$ value \\
\hline \multirow[t]{8}{*}{ All-cause mortality } & $\begin{array}{l}\text { HOMA-IR } \\
\text { (per SD increase) }\end{array}$ & 4,742 & 1,943 & 0.94 & $0.89,0.98$ & 0.009 & 1.00 & $0.94,1.06$ & 0.88 \\
\hline & HOMA-IR T1 & 1,766 & 759 & Ref & - & & Ref & - & \\
\hline & HOMA-IR T2 & 1,675 & 655 & 0.86 & $0.77,0.95$ & & 0.92 & $0.82,1.03$ & \\
\hline & HOMA-IR T3 & 1,301 & 529 & 0.89 & $0.80,1.00$ & 0.010 & 1.02 & $0.90,1.17$ & 0.14 \\
\hline & $\begin{array}{l}\text { Insulin } \\
\text { (per SD increase) }\end{array}$ & 4,923 & 2,034 & 0.93 & $0.89,0.98$ & 0.003 & 0.99 & $0.94,1.05$ & 0.76 \\
\hline & Insulin T1 & 1,745 & 763 & Ref & - & & Ref & - & \\
\hline & Insulin T2 & 1,674 & 661 & 0.86 & $0.78,0.96$ & & 0.93 & $0.83,1.04$ & \\
\hline & Insulin T3 & 1,504 & 610 & 0.90 & $0.80,1.00$ & 0.015 & 1.03 & $0.91,1.17$ & 0.16 \\
\hline \multirow[t]{8}{*}{ CHD mortality } & $\begin{array}{l}\text { HOMA-IR } \\
\text { (per SD increase) }\end{array}$ & 4,742 & 470 & 0.97 & $0.88,1.07$ & & 0.96 & $0.86,1.08$ & 0.54 \\
\hline & HOMA-IR T1 & 1,766 & 167 & Ref & - & & Ref & - & \\
\hline & HOMA-IR T2 & 1,675 & 178 & 1.10 & $0.89,1.35$ & & 1.12 & $0.90,1.41$ & \\
\hline & HOMA-IR T3 & 1,301 & 125 & 1.00 & $0.79,1.26$ & 0.63 & 1.03 & $0.79,1.36$ & 0.56 \\
\hline & $\begin{array}{l}\text { Insulin } \\
\text { (per SD increase) }\end{array}$ & 4,923 & 494 & 0.97 & $0.88,1.06$ & 0.46 & 0.97 & $0.87,1.08$ & 0.57 \\
\hline & Insulin T1 & 1,745 & 170 & Ref & - & & Ref & - & \\
\hline & Insulin $\mathrm{T} 2$ & 1,674 & 166 & 1.00 & $0.81,1.24$ & & 1.04 & $0.83,1.30$ & \\
\hline & Insulin T3 & 1,504 & 158 & 1.07 & $0.86,1.33$ & 0.77 & 1.15 & $0.89,1.48$ & 0.54 \\
\hline \multirow[t]{8}{*}{ All CVD (Scottish cohort only) } & $\begin{array}{l}\text { HOMA-IR } \\
\text { (per SD increase) }\end{array}$ & 2,039 & 590 & 1.00 & $0.91,1.09$ & 0.99 & 1.01 & $0.91,1.12$ & 0.90 \\
\hline & HOMA-IR T1 & 668 & 185 & Ref & - & & Ref & - & \\
\hline & HOMA-IR T2 & 768 & 238 & 1.08 & $0.89,1.31$ & & 1.06 & $0.87,1.30$ & \\
\hline & HOMA-IR T3 & 603 & 167 & 0.95 & $0.77,1.17$ & 0.42 & 0.94 & $0.74,1.20$ & 0.50 \\
\hline & $\begin{array}{l}\text { Insulin } \\
\text { (per SD increase) }\end{array}$ & 2,191 & 644 & 0.99 & $0.91,1.08$ & 0.82 & 0.99 & $0.90,1.09$ & 0.89 \\
\hline & Insulin T1 & 754 & 213 & Ref & - & & Ref & - & \\
\hline & Insulin T2 & 782 & 245 & 1.09 & $0.90,1.31$ & & 1.09 & $0.90,1.32$ & \\
\hline & Insulin T3 & 655 & 186 & 0.97 & $0.80,1.18$ & 0.47 & 0.98 & $0.78,1.23$ & 0.50 \\
\hline
\end{tabular}

$p$ value is for test for linear trend in HR estimates (across distribution)

Model 1 adjusted for country, randomised treatment

Model 2 additionally adjusted for age, sex, smoking status, BMI, systolic blood pressure, diastolic blood pressure, HDL-cholesterol, LDL-cholesterol, $\log$ triacylglycerols, histories of hypertension, coronary, cerebrovascular and peripheral vascular disease, diuretics, ACE inhibitors, angiotensin receptor blockers, $\beta$-blockers and calcium channel blockers

T1, lower third of the distribution; T2, middle third of the distribution; T3, top third of the distribution 
HOMA-IR nor fasting insulin showed any trend towards an association either in minimally or fully adjusted models (Table 3 and Fig. 2). These results were consistent when the data were modelled using exposures as continuous variables to maximise statistical power (Table 3).

When effect estimates were stratified by within-trial follow-up versus post-trial follow-up, HRs were similar in both strata. There was also no strong evidence of interaction for the effect of insulin resistance by any covariate in the fully adjusted model for any endpoint (ESM Fig. 2 - ESM Fig. 4).

\section{Discussion}

During long-term follow-up of the PROSPER cohort of older people without diabetes, we observed no compelling evidence of an association of HOMA-IR with all-cause mortality, CHD mortality or CVD events over 8.6 years. By contrast, both fasting insulin and HOMA-IR were powerfully associated with risk for developing diabetes within the 3.2 years of intrial follow-up, providing evidence of internal validity. Our results therefore suggest that insulin and insulin resistance are unlikely to be important CVD risk factors in elderly people without diabetes $[6,14,15]$. This is an important finding given that older people have been relatively poorly represented in studies to date but are an increasingly important group in public health terms. This is also consistent with a body of work across age ranges showing that many risk factors for CVD and diabetes are actually somewhat distinct [2-5]. Our work shows that, as with metabolic syndrome in the elderly [2], insulin resistance is a strong predictor of diabetes but has minimal prediction and therefore potential clinical relevance to vascular outcomes in the elderly.

Two recent meta-analyses have investigated the extent of the associations between insulin and HOMA-IR, and CVD [8, 9]. A study-level meta-analysis showed modest borderline significant associations between fasting insulin and CHD in those without baseline diabetes (OR comparing extreme thirds of the distribution was 1.12 [95\% CI $0.98,1.28]$, with moderate heterogeneity; $\mathrm{I}^{2}$ 59\%) [8]. A more recent meta-analysis in people without diabetes showed, by comparing pooled standardised relative risks, that one standard deviation higher insulin (16 studies) and HOMA-IR (17 studies) were only modestly associated with CVD outcomes: risk ratios 1.13 (95\% CI 1.05,1.22) (I $\left.\mathrm{I}^{2} 58.3 \%\right)$ and 1.25 (95\% CI 1.16,1.35) $\left(\mathrm{I}^{2} 52.4 \%\right)$, respectively. Again, both meta-analyses demonstrated moderate heterogeneity. Our results in the elderly meaningfully extend these findings in a single powerful study including 1,943 all-cause mortality events and 470 CHD deaths.

The observational nature of this study means that causal inference (or inference of lack of causation) must be made with caution, although emerging data also support our results by lending at best weak support for a causal role of insulin resistance in CVD. Recent data from the Framingham Heart Study show that genetic variants near the IRS1 locus (associated with insulin resistance) are not associated with subclinical atherosclerosis [16]. Other recent trial evidence from the CAMERA randomised placebo-controlled trial of metformin in patients with CHD showed no reduction in carotid intimamedia thickness with metformin compared with placebo [17],
Fig. 2 Association of thirds of insulin and HOMA-IR with risk of endpoints. Diamonds refer to the point estimate for the HR comparing the middle third (open diamonds) or top third (black diamonds) with the bottom third of the distribution. Bars represent $95 \% \mathrm{CI} ; x$-axis on the log scale

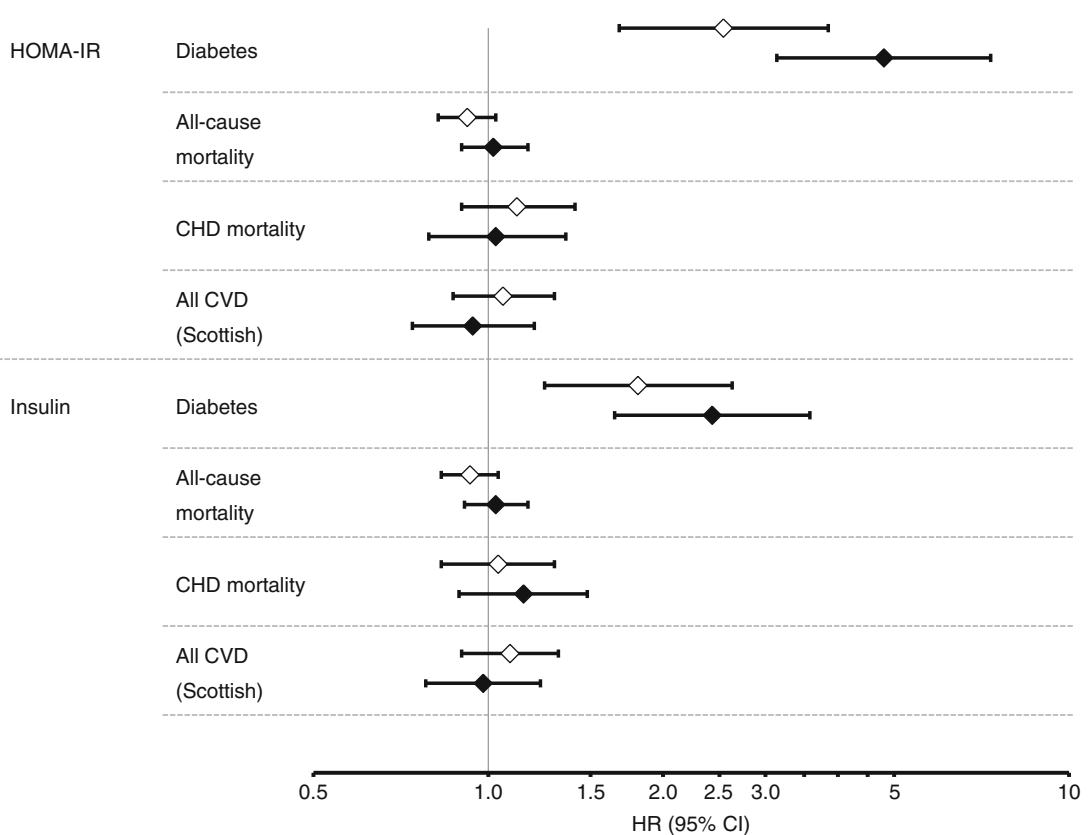


and metformin for 4 months also had no benefit on left ventricular function in patients after ST segment elevation myocardial infarction in another recent trial [18].

Fasting insulin and HOMA-IR are well-accepted surrogate measures of insulin resistance [19, 20], but are not gold standard measures of whole body insulin resistance, which is obtained by euglycaemic-hyperinsulinaemic clamping. It is a limitation of our study that we did not have such measures, although no large cohorts with prospective data that we are aware of have data on both insulin sensitivity measured by clamping techniques and sufficient numbers of CVD events to allow adequately powered statistical analysis. The multinational Relationship between Insulin Sensitivity and Cardiovascular Disease (RISC) Study recently reported weak cross-sectional associations of insulin sensitivity (measured by euglycaemic clamps) with carotid intima-media thickness in men but not in women, and, potentially more importantly, insulin sensitivity was not associated with 3 year changes in carotid intima-media thickness [21]. We also did not measure model-based insulin sensitivity or use data from OGTT; a recent meta-analysis suggests that correlation with euglycaemic-clamp-derived insulin sensitivity is $r=-0.60$ for $\log$ HOMA-IR, $r=0.57$ for model-based insulin sensitivity, and around $r=0.70$ for various OGTT-based measures [22]. An accompanying editorial suggests 'Where the focus is solely on insulin resistance...surrogate markers based on fasting samples alone are as valid as those that require multiple samples and an oral glucose load' [23].

Further potential limitations of the study require consideration. It is possible that our results may be influenced by the age of the PROSPER cohort; some risk factors are less strongly associated with CVD in older people. Although elderly, the PROSPER cohort represents a group of people in good cognitive health, and many other risk factors such as inflammatory markers have shown expected associations with CVD risk in this population $[13,24]$, and of course we show that insulin resistance strongly predicts diabetes. Older people are also an increasingly important age group in terms of public health and population CVD burden. It is also possible that insulin levels would be associated with increased cardiovascular risk over an even longer follow-up (than the 8.6 years studied here) given the period required for conversion into diabetes and then for those who have developed diabetes to accumulate CVD risk. However, this would suggest that diabetes is a CVD risk factor (i.e. hyperglycaemia per se), and not necessarily insulin resistance in the elderly without diabetes, and of course, diabetic patients also lose pancreatic insulin secretory capacity. Lack of availability of data on non-fatal CVD in the Irish and Dutch PROSPER participants during the extended follow-up limited the power of these analyses. Regardless, PROSPER remains one of the most powerful prospective studies relating HOMA-IR to CVD and is one of the very few to simultaneously relate it to incident diabetes.

In conclusion, our data support insulin resistance as a predictor of diabetes in later life but, perhaps surprisingly, suggest this pathway is of minimal/negligible importance to CVD outcomes in the elderly.

Acknowledgements We thank L. Cherry (Institute of Cardiovascular and Medical Sciences, University of Glasgow, Glasgow, UK) for technical assistance.

Funding The study was supported by the Chief Scientist Office of the Scottish Executive Health Department who provided funding for longterm follow-up of the Scottish cohort: CZH/4/637. PW is supported by BHF fellowship FS/12/62/29889.

Duality of interest The authors declare that there is no duality of interest associated with this manuscript.

Contribution statement AJC, JWJ, RGW, BMB, PMK, AB, DJS, IF and NS were involved in study design and conception. PW, DP and NS were involved in data acquisition. SML and IF conducted data analysis. PW, DP and NS wrote the first draft of the manuscript. SML, AJC, JWJ, RGW, BMB, PMK, AB, DJS and IF reviewed the manuscript for important intellectual content. All authors approved the final version for publication. NS and PW are responsible for the integrity of the work as a whole.

\section{References}

1. Haffner SM, Lehto S, Ronnemaa T, Pyorala K, Laakso M (1998) Mortality from coronary heart disease in subjects with type 2 diabetes and in nondiabetic subjects with and without prior myocardial infarction. N Engl J Med 339:229-234

2. Sattar N, McConnachie A, Shaper AG et al (2008) Can metabolic syndrome usefully predict cardiovascular disease and diabetes? Outcome data from two prospective studies. Lancet 371:1927-1935

3. Preiss D, Welsh P, Murray HM et al (2010) Fasting plasma glucose in non-diabetic participants and the risk for incident cardiovascular events, diabetes, and mortality: results from WOSCOPS 15-year follow-up. Eur Heart J 31:1230-1236

4. Welsh P, Murray HM, Buckley BM et al (2009) Leptin predicts diabetes but not cardiovascular disease: results from a large prospective study in an elderly population. Diabetes Care 32:308-310

5. Emerging Risk Factors Collaboration (2010) Diabetes mellitus, fasting blood glucose concentration, and risk of vascular disease: a collaborative meta-analysis of 102 prospective studies. Lancet 375: 2215-2222

6. Tsuchiya K, Tanaka J, Shuiqing Y et al (2012) FoxOs integrate pleiotropic actions of insulin in vascular endothelium to protect mice from atherosclerosis. Cell Metab 15:372-381

7. DeFronzo RA (2010) Insulin resistance, lipotoxicity, type 2 diabetes and atherosclerosis: the missing links. The Claude Bernard Lecture 2009. Diabetologia 53:1270-1287

8. Sarwar N, Sattar N, Gudnason V, Danesh J (2007) Circulating concentrations of insulin markers and coronary heart disease: a quantitative review of 19 Western prospective studies. Eur Heart J 28:2491-2497 
9. Gast KB, Tjeerdema N, Stijnen T, Smit JW, Dekkers OM (2012) Insulin resistance and risk of incident cardiovascular events in adults without diabetes: meta-analysis. PLoS ONE 7:e52036

10. Shepherd J, Blauw GJ, Murphy MB et al (2002) PROspective Study of Pravastatin in the Elderly at Risk. Pravastatin in elderly individuals at risk of vascular disease (PROSPER): a randomised controlled trial. Lancet 360:1623-1630

11. Lloyd SM, Stott DJ, de Craen AJ et al (2013) Long-term effects of statin treatment in elderly people: extended follow-up of the PROspective Study of Pravastatin in the Elderly at Risk (PROSPER). PLoS ONE 8:e72642

12. Shepherd J, Blauw GJ, Murphy MB et al (1999) The design of a prospective study of Pravastatin in the Elderly at Risk (PROSPER). PROSPER Study Group. Am J Cardiol 84:1192-1197

13. Sattar N, Murray HM, Welsh P et al (2009) Are markers of inflammation more strongly associated with risk for fatal than for nonfatal vascular events? PLoS Med 6:e1000099

14. Reddy KJ, Singh M, Bangit JR, Batsell RR (2010) The role of insulin resistance in the pathogenesis of atherosclerotic cardiovascular disease: an updated review. J Cardiovasc Med 11:633-647

15. Ginsberg HN (2000) Insulin resistance and cardiovascular disease. $\mathrm{J}$ Clin Invest 106:453-458

16. Lim S, Hong J, Liu CT et al (2013) Common variants in and near IRS1 and subclinical cardiovascular disease in the Framingham Heart Study. Atherosclerosis 29:149-154

17. Preiss D, Lloyd S, Ford I et al (2014) Metformin for non-diabetic patients with coronary heart disease (the CAMERA study): a randomised controlled trial. Lancet Diabetes Endocrinol 2:116124

18. Lexis CP, van der Horst IC, Lipsic E et al (2014) Effect of metformin on left ventricular function after acute myocardial infarction in patients without diabetes: the GIPS-III randomized clinical trial. JAMA 311:1526-1535

19. Hanley AJ, Williams K, Gonzalez C et al (2003) Insulin Resistance Atherosclerosis Study. Prediction of type 2 diabetes using simple measures of insulin resistance: combined results from the San Antonio Heart Study, the Mexico City Diabetes Study, and the Insulin Resistance Atherosclerosis Study. Diabetes 52:463-469

20. Bonora E, Targher G, Alberiche $\mathrm{M}$ et al (2000) Homeostasis model assessment closely mirrors the glucose clamp technique in the assessment of insulin sensitivity: studies in subjects with various degrees of glucose tolerance and insulin sensitivity. Diabetes Care 23:57-63

21. Kozakova M, Natali A, Dekker J (2013) Insulin sensitivity and carotid intima-media thickness: relationship between insulin sensitivity and cardiovascular risk study. Arterioscler Thromb Vasc Biol 33:1409-1417

22. Otten J, Ahrén B, Olsson T (2014) Surrogate measures of insulin sensitivity vs the hyperinsulinaemic-euglycaemic clamp: a metaanalysis. Diabetologia 57:1781-1788

23. Petrie JR (2014) Evidence-based estimation of insulin resistance. Diabetologia 57:1743-1745

24. Welsh P, Murray HM, Ford I et al (2011) Circulating interleukin-10 and risk of cardiovascular events: a prospective study in the elderly at risk. Arterioscler Thromb Vasc Biol 31:2338-2344 